\title{
Application of Support Vector Machines Arithmetic in Body Posture Detection
}

\author{
Bo $\mathrm{Du}^{1, \mathrm{a}^{*}}$, Lu Yin ${ }^{2, \mathrm{~b}}$ and Zhuo Zhang ${ }^{1, \mathrm{c}}$ \\ ${ }^{1}$ Changchun Institute of Technology, Changchun 130012,China \\ ${ }^{2}$ QiMing Information Technology Co.,Ltd , Changchun 130012,China \\ a15347158@qq.com, ${ }^{\mathrm{b}}$ 270941298@qq.com, ${ }^{\mathrm{c} 20894321 @ q q . c o m}$
}

\section{Keywords: Support Vector Machines STM32F103C8T6 SMV SMA Fall}

Abstract. It is very critical to detect elder people's falling down in time for it will be harmed to them even to death if they can't be found early.

Body posture detection system is based on STM32F103C8T6 as controller which can detect falling down in time from the two parts of host and slave. Data transferred by Bluetooth between host and slave of this system. The slave detect human movement data by MPU6050, the host analysis and display the current human body posture.

A lot of testing data shows that it can get higher accuracy from using Support Vector Machine (SVM) algorithm to posture analysis, integration of acceleration signal, analysis of Signal Magnitude Vector(SMV) and Signal Magnitude Area(SMA) signals, falling and posture judgement from the assistant of intersection angle that between body and ground on the vertical direction.

\section{Introduction}

According to China's 2010 national census data show, the number of people over the age of 60 to 1.78 billion, accounted for $13 \%$ of the total population, the number of people over the age of 65 to 1.19 billion, accounted for $8.87 \%$ of the total number of the country. From the data analysis, we can know that the structure of population data in China is aging.

Research shows that among the people over age of 65 , nearly $30 \%$ of them who have fallen once or more every year, and among the people over age of 80 , more than $50 \%$ of them who have fallen at least once every year. And this fall will cause more serious damage with the increase of age. Many serious consequences attribute to can't find and relief in time after falling down rather than directly fall down. So it is very significant to find and alarm for the old man's falling down in time.

System Composition. Body posture detection system is divided into host and slave. Host is composed of controller, display module and wireless transmission module. Slave is composed of controller, sensor and wireless transmission module. Slave collects human body movement signals by the sensors, and transfers the signals by the wireless transmission module to host. Host receives signals by the wireless transmission from slave, judges moving posture by data analysis and processing, and has display and alarm function. Body posture detection structure chart is shown in Fig.1.

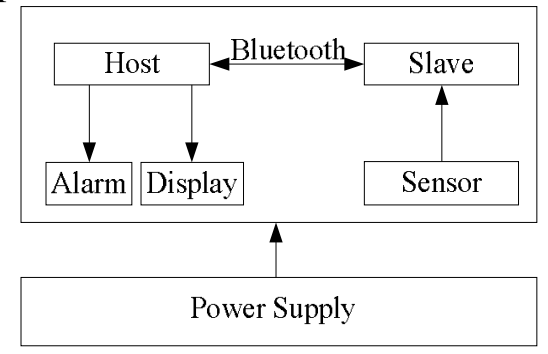

Fig. 1 Body posture detection structure chart

\section{Hardware Design.}


Controller. STM32F103 series is the latest ARM processor core based on ARMv7 architecture, with high performance, low cost, low power consumption characteristics.STM32F103C8T6 is a 32 bit microcontroller based on the Cortex-M3 kernel. When using the external crystal oscillator, the maximum main frequency of the internal is $72 \mathrm{MHz}$, using the internal crystal oscillator, the maximum main frequency is $64 \mathrm{MHz}$. STM32F103C8T6 has 64KB flash memory and 20KB SRAM.

Detection Circuit. Body posture detection system uses MPU6050 sensor (three axis gyroscope + three axis acceleration), the acceleration and angular velocity of the three axes can be measured. MPU6050 module itself has voltage stabilizing circuit, compatible with $3.3 \mathrm{~V} / 5 \mathrm{~V}$ system ${ }^{[1]}$.MPU6050 angular velocity measurement range is $\pm 250 、 \pm 500 、 \pm 1000$ and $\pm 2000 \%$ sec, accelerator measurement range is $\pm 2 \mathrm{~g}, \pm 4 \mathrm{~g}, \pm 8 \mathrm{~g}$ and $\pm 16 \mathrm{~g}$, with the highest to $400 \mathrm{kHz}$ I2C or the highest to 20 MHz SPI interface.STM32F103C8T6 completes data communication with MPU6050 by serial port. MPU6050 and STM32F103C8T6 interface circuit diagram is shown in Fig.2(a).

Display and Alarm Circuit. Display uses Organic Light-Emitting Diode, also known as Organic Electroluminesence Display(OELD). With energy saving, light and other characteristics, since 2007, the life has been greatly improved, with many LCD incomparable advantages. Alarm uses the buzzer. OLED interface circuit diagram is shown in Fig.2(b), Alarm interface circuit diagram is shown in Fig.2(c).

Wireless Communication Circuit. Wireless communication using BT-HC-05 Bluetooth module, Clearing effective distance is 10 meters (power level for CLASS 2). Bluetooth can be full duplex serial port to use after pairing, no need to know any Bluetooth protocol, support 8 data bits and 1 stop bit, can set the odd-even check communication format, support the standard baud rate 4800bps--1382400bps. Bluetooth interface circuit diagram is shown in Fig.2(d).

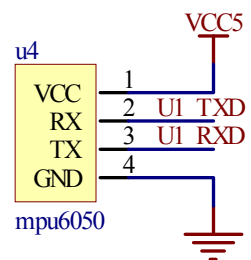

(a) MPU6050

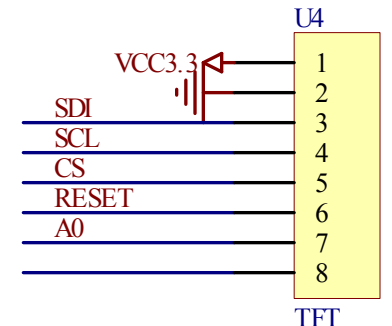

(b) OLED

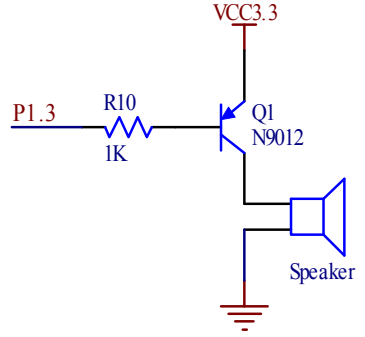

(c) Alarm

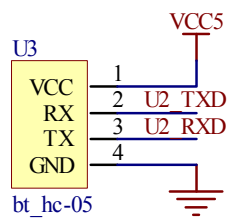

(d) Bluetooth

Fig. 2 interface circuit diagram

\section{Software Design.}

Coordinate Establishment. When the human body falls, the body's acceleration, speed, angle will change accordingly. Therefore, the acceleration and angular velocity are collected and processed by the appropriate algorithm, so as to judge the posture and find the phenomenon of falling down timely.

After multiple measurement, it can be found that the change of acceleration signal can reflect the change of human motion, when sensor worn on waist axis of the body. The sensor has three axes, $\mathrm{X}$-axis, $\mathrm{Y}$-axis, and $\mathrm{Z}$-axis, and the people who stand upright, the $+\mathrm{Z}$ is pointing upward, the $+\mathrm{Y}$ is pointing to the left and parallel to the ground, and the $+X$ points to the front and parallel to the ground. Three-dimensional direction schematic diagram is shown in Fig.3. Coordinate schematic diagram is shown in Fig.4.
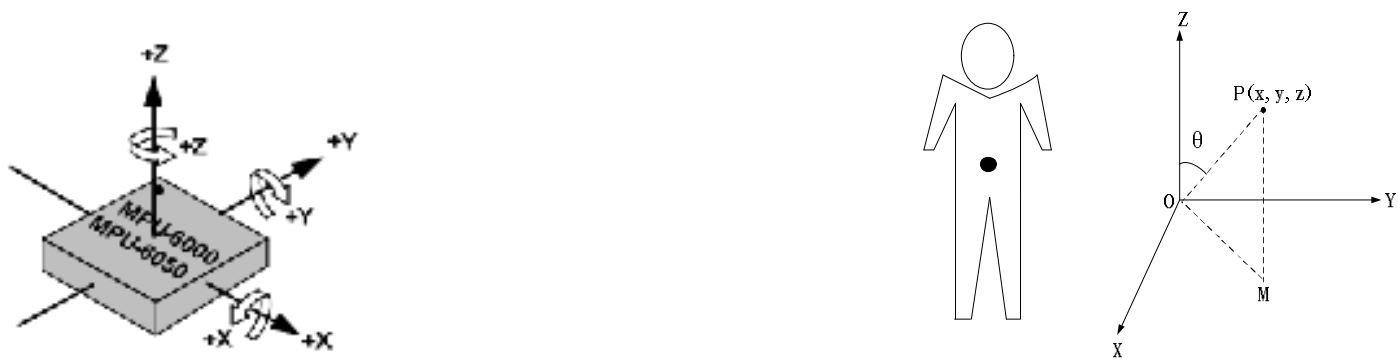
Fig.3 Three-dimensional direction schematic diagram

Fig.4 Coordinate schematic diagram

The detection state of detection system is mainly aimed at the human body standing, walking, brisk walking, sit down and fall down.

Support Vector Machines Arithmetic. Slave collects data, and transfers it to host by Bluetooth. Host filtering the receive data first, then uses Support Vector Machine arithmetic to process data.

Support Vector Machine (SVM) in solving small sample, nonlinear and high dimensional pattern recognition show many unique advantages, and can be applied to other machine learning problems such as function fitting. The so-called Support Vector is the training sample points on the edge of the interval. Here's "machine" is actually an algorithm. In machine learning field, the algorithm is often regarded as a machine. Support Vector Machine (SVM) is similar to the neural network, it is a learning mechanism, but it is different from the neural network, SVM uses mathematical methods and optimization techniques.

The signals of human body movement are collected and analyzed. It is found that the acceleration signal is closely related to human body movement and can obviously reflect the movement of human body. Therefore, the acceleration signal is integrated and analyzed, the angle signal is auxiliary.

ax, ay and az are three axis acceleration signals, and Signal Magnitude Vector (SMV) reflects the intensity of the movement of the human body. SMV value will increase rapidly when the falls occur. Select the appropriate threshold, SMV can distinguish the human body movement to a certain extent. SMV value is shown in table 1.

$$
\operatorname{SMV}=\sqrt{\mathrm{a}_{\mathrm{x}}^{2}+\mathrm{a}_{\mathrm{y}}^{2}+\mathrm{a}_{\mathrm{z}}^{2}}
$$

Table 1 SMV value

\begin{tabular}{|c|c|c|c|c|c|}
\hline Posture & Stand & Walk & brisk walking & sit down & $\begin{array}{c}\text { fall } \\
\text { down }\end{array}$ \\
\hline $\mathrm{SMV}=\sqrt{\mathrm{A}_{\mathrm{X}}^{2}+\mathrm{A}_{\mathrm{Y}}^{2}+\mathrm{A}_{\mathrm{Z}}^{2}}$ & $1.03 \mathrm{~g}$ & $\begin{array}{c}1.17 \mathrm{~g}--1.25 \\
\mathrm{~g}\end{array}$ & $1.27 \mathrm{~g}--1.45 \mathrm{~g}$ & $\begin{array}{c}1.5 \mathrm{~g}--1.6 \\
\mathrm{~g}\end{array}$ & $\begin{array}{c}1.87 \mathrm{~g}--2 . \\
4 \mathrm{~g}\end{array}$ \\
\hline
\end{tabular}

Note: The conner is a man,22 years old, height $180 \mathrm{~cm}$, and weight $66 \mathrm{~kg}$

But some of the larger movement of non-fall action can also cause larger SMV, such as brisk walking, running, sit down, etc. While faint is a slow fall, it's SMV is not big. These two cases can cause false judgment. Therefore, the use of Signal Magnitude Area (SMA) for further analysis and judgment.

$$
\mathrm{SMA}=\frac{1}{\mathrm{k}}\left(\sum_{i=1}^{k}\left|a_{x i}\right|+\sum_{i=1}^{k}\left|a_{y i}\right|+\sum_{i=1}^{k}\left|a_{z i}\right|\right)
$$

The SMA is the accumulation of the acceleration signal in a period of time, which can effectively remove the false judgment. SMA can not reflect the effect, if accumulation time is too short.SMA will mistakenly increase, because waveform contains two or more acceleration peak, if accumulation time is too long. So the time window setting is the key. Because the highest frequency of human body movement not exceed $20 \mathrm{~Hz}$, the system sample frequency is set to $50 \mathrm{~Hz}$, the time window is set to1s.After test analysis, it is considered non-strenuous exercise, when SMA is less than $1.4 \mathrm{~g}$. It is considered strenuous exercise or falls, when SMA is more than or equal to $1.4 \mathrm{~g}$.

When a fall occurs, the angle between the body and the vertical direction of the ground is greater than $60^{\circ}$, close to $90^{\circ}$. Therefore, this angle is an auxiliary judgment condition for falls.

$$
\theta=\arctan \left(\frac{\sqrt{a_{x}^{2}+a_{y}^{2}}}{a_{z}}\right)
$$

SMV, SMA and $\theta$ are as the condition of judgment, to judge whether a fall occurs. The system will carry on the posture judgment, if no fall occurs.

Software Process. Body posture detection system is divided into host and slave. Host shows the human body posture, and judges whether a fall occurs. Slave collects human body movement signals, 
and transfers the signals by Bluetooth to host. Host and slave program flow charts are shown in Fig.5 and Fig.6.

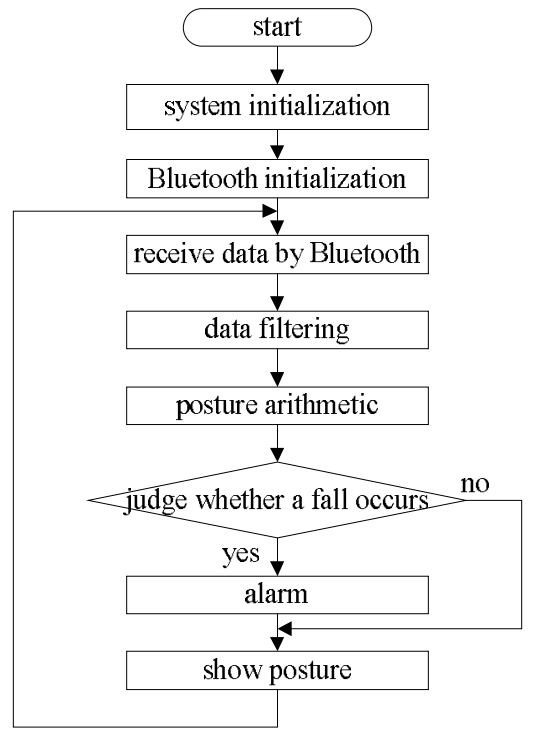

Fig. 5 Host program flow chart

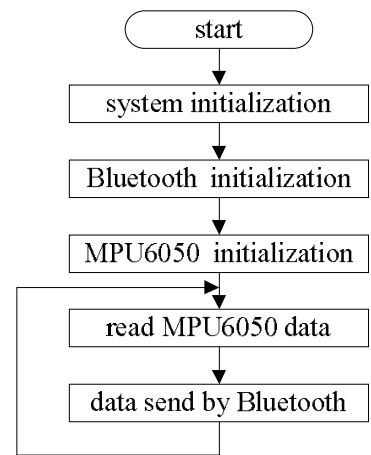

Fig. 6 Slave program flow chart

System debug and Conclusion. To debug the system, when the design of hardware circuit and the software algorithm has been completed. Human posture displays are as shown in Fig.7, Fig.8, Fig.9.

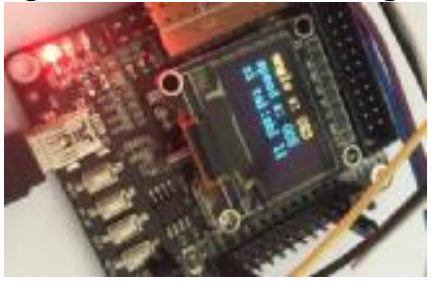

Fig.7 Stand display graph

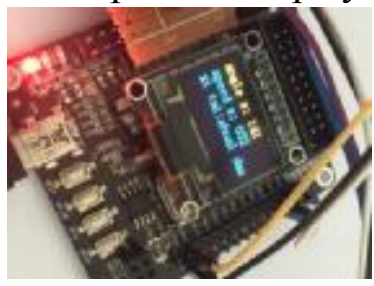

Fig. 8 Fall display graph

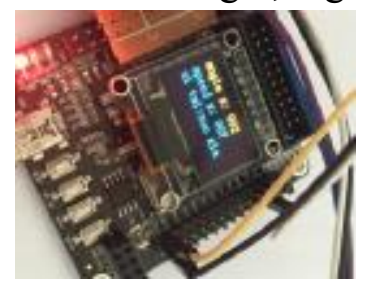

Fig. 9 Sit down display graph

Body posture detection system is based on STM32F103C8T6, uses MPU6050 to collect the acceleration signal of human body motion. The system can judge posture and detect falling accident, by support vector machine (SVM) algorithm for posture analysis. It can display the posture information on OLED, and transfer data via Bluetooth. After testing and analysis, the system can judge the posture, and detect falling accident timely in order to gain the more subsequent rescue time for the elderly.

Acknowledgements. This paper is supported by the Jilin Province Education Department Science and technology research project: 120140059, 12014K013.

\section{References}

[1] Junjian Zhang.Study on the detection of body position mutation based on the three axis acceleration sensor [D].Shandong Normal University,2014. In Chinese

[2] McEwen J, Wiaux Y. A Novel Sampling Theorem on the Sphere[J].IEEE Transactions on Signal Processing. 2011,59(12):7876-5887.

[3] Tuanshan Zhang,Ying He. The Kalman filter application of pose detection of two wheeled self balancing scooter [J]. MCU and embedded system application,2014,(5):33-35. In Chinese

[4] Yan $\mathrm{Zhu}$, Wei Fu. The pose estimation algorithm of the four rotor unmanned aerial vehicle based on Kalman filter without trace [J]. Journal of testing technology, 2014,28(3):194-197. In Chinese

[5] Xinxiang Sun. Research and application of fall detection technology based on three axis accelerometer [D]. Shanghai Jiao Tong University,2008. In Chinese

[6] InvenSense Inc. MPU-6000 and MPU-6050 Product Specification Revision 3.2.pdf. www.invensense.com, 2011:7. 
[7] Ting Wang, Wenwu Feng, Dechao Luo. Application of wireless sensor in the gesture measuring of human body joint motion[J].Techniques of Automation and Application,2009,28(8):84.In Chinese 